\title{
Drugs in preclinical and early stage clinical development for pancreatic cancer
}

\author{
Swapna Asuthkar ${ }^{1}$, Jasti S. Rao ${ }^{1,2}$, Christopher S Gondi ${ }^{{ }^{*}}$ \\ Departments of Cancer Biology \& Pharmacology, ${ }^{1}$ and Neurosurgery, ${ }^{2}$ University of Illinois College of \\ Medicine at Peoria, One Illini Drive, Peoria, IL 61605, USA
}

Swapna Asuthkar, Ph.D.

Post-doctoral Research Associate

University of Illinois College of Medicine

Department of Cancer Biology and Pharmacology

One Illini Drive

Peoria, IL 61605

309-671-3445 - phone

309-671-3442 - fax

asuthkar@uic,edu - e-mail

Jasti S. Rao, Ph.D.

Priofessor and Head

University of Illinois College of Medicine

Department of Cancer Biology and Pharmacology

One Illini Drive

Peoria, IL 61605

309-671-3445 - phone

309-671-3442 - fax

jsrao@uic.edu

Christopher S. Gondi, Ph.D.* (corresponding author)

Research Assistant Professor

University of Illinois College of Medicine

Department of Cancer Biology and Pharmacology

One Illini Drive

Peoria, IL 61605

309-671-3445 - phone

309-671-3442 - fax

gondi@uic,edu - e-mail

Declaration of interest: The authors state no conflict of interest and have received no payment in preparation of this manuscript. 


\begin{abstract}
Introduction: Pancreatic cancer (PC) is the fourth leading cause of cancer-related deaths in the U.S. and Europe and the lethality of this cancer is demonstrated by the fact that the annual incidences are approximately equal to the annual deaths. Current therapy for PC is multimodal, involving surgery and chemotherapy. Clinical symptoms are unspecific, and consequently about 85\% of patients with PC are diagnosed at advanced tumor stages without any surgical therapy options. Since the therapeutic rates for PC are so dismal, it is essential to review the clinical targets for diagnosis and treatment of this lethal cancer.
\end{abstract}

Areas covered: In this review, we discuss potential treatment options for PC by identifying molecular targets including those involved in cell proliferation, survival, migration, invasion and angiogenesis. Targeting these molecules in combination with surgery could improve the clinical outcome for PC patients.

Experts opininon: For a decade, gemcitabine has remained the single first-line chemotherapeutic agent for advanced adenocarcinoma of the pancreas, however, less than 25\% of patients benefit from gemcitabine. The reason for frequent reoccurrence of PC after conventional methods such as surgery, radiation and/or chemotherapy is due to the lack of understanding of the basic underlying metabolic cause of the cancer and thus consequently remains uncorrected. Our understanding of drug resistance in PC is still not clear and may be answered by focusing on new useful biomarkers and their role in chemo- and radioresistance.

Key words: pancreatic cancer (PC), gemcitabine, chemo sensitivity, molecular targets. 


\section{Introduction}

Pancreatic cancer (PC) is an aggressive disease that has assumed a growing importance because of its increasing incidence and poor prognosis. Every year, around 170,000 new cases of PC occur worldwide, out of which more than 42,000 cases are in the U.S., (ACS) [1-3] The lethality of this malignancy is demonstrated by the fact that the annual incidence is approximately equal to the annual deaths and is the fourth leading cause of cancer-related deaths in the U.S. and Europe and despite advances in clinical management, the 6-month progressionfree survival remains below 15\% [4].

Prior research has shown that the causes of PC include smoking [5], chronic pancreatitis [6], and genetic predispositions [7,8]. Smoking is implicated in 25-30\% of PC patients. The association of obesity and diabetes to PC is about 30\% [9]. A number of hereditary syndromes are also known to be correlated with incidence of PC; around $4-16 \%$ of cases appear to be familial [10-12]. Some of the known hereditary syndromes associated with PC include familial atypical multiple mole melanoma syndrome (FAMM), Peutz-Jeghers syndrome, BRCA2 mutations, hereditary nonpolyposis colorectal cancer syndrome (HNPCC), Lynch syndrome and hereditary pancreatitis [13-17]. Interestingly, a number of acquired or somatic mutations have been identified with high frequencies in human PC specimens. These include k-ras mutations in $>90 \%$ of PC specimens [18] and p16, p53 and DPC4 tumor suppressor gene mutations [19,20]. The p16 tumor suppressor gene is a regulator of cell-cycle control and may be inactivated in 98\% of human PC specimens. These findings not only provide insight into the genetic etiology of pancreatic tumors, but also highlight potential molecular targets for therapeutic interventions. Studies on global trends show that the rate of PC seems to be inversely proportional to solar 
radiation in other words; increasing risk of PC is associated with decrease in the levels of vitamin D [21,22]. This explains the fact that PC rates are four times higher in northern countries than in countries located closer to the equator such as Egypt, Tunisia, Zimbabwe and India [22,23].

Around $80-90 \%$ of PC cases are diagnosed in the non-resectable stage with the presence of widespread local disease or metastasis and thereby contributing to a very low survival rate [24]. There are several factors that contribute to the high mortality rate associated with PC. Primarily, early detection, standardized screening and diagnosis are lacking for PC. While tests such as mammograms, colonoscopy, and PSA are available that can detect precancerous cells or biomarkers of breast, colorectal and prostate cancer, such pre-screening tests are not as readily available or accessible in the case of PC. This is one of the major reasons that screening for PC has never become routine, but instead the disease is detected only in its later stages when it is almost uniformly fatal. Secondly, PC is one of the most drug-resistant cancers [25], making it very difficult to treat. Finally, there is a high rate of relapse, even in patients with early stage disease who receive adjuvant therapy [26]. Since the therapeutic rates for PC are so dismal, it is essential to review the clinical targets for diagnosis and treatment of this lethal cancer.

\section{Current status of treatment options for PC}

Invasive surgery may be an option for PC patients and is usually followed by chemotherapy and/or radiation. A surgical procedure called the Whipple procedure or pancreatoduodenectomy involves removing the head of pancreas, a portion of small intestine duodenum, gallbladder and a part of the bile duct. Whipple surgery carries a risk of infection and bleeding accompanied with delayed gastric emptying [27]. Another surgical procedure called distal pancreatectomy involves the removal of tumors in the tail of the pancreas. Surgery is an invasive procedure and carries a 
risk of bleeding and infection [27]. Despite surgery with curative intent, the five-year survival rates for these patients remains close to $20 \%$ or less, indicating that even patients with localized small cancers $(2 \mathrm{~cm})$ with no lymph node metastases are likely to die of metastatic PC [28]. Therefore, improvements in therapeutic options that may serve as adjuncts to surgery and systemic treatments for advanced PC are the need of the hour and will provide the greatest hope of improving the clinical outcomes in this disease.

Adjuvant therapy is post-operative chemotherapy or radiotherapy or both, administered to patients with no detectable evidence of residual disease but who are likely to harbor microscopic tumor deposits that if left untreated, will lead to tumor recurrence and death. In one randomized trial conducted by the Gastrointestinal Tumor Study Group (GITSG), 43 patients were studied and randomized to surgery alone, or surgery followed by 24 months of 5-fluorouracil (5-FU)based chemoradiotherapy. This study found an improvement in median survival from 19 to 24.5 months, however this study was not statistically significant $(\mathrm{P}=0.208)$ [29]. The benefits of chemoradiation have been questioned since the publication of the European Study Group for Pancreatic cancer (ESPAC) 1 trial [30,31] which suggested that adjuvant chemoradiotherapy might even harm patients after a curative surgical resection. This was the largest adjuvant trial involving 548 patients of PC with 2 X 2 factorial designs wherein the patients after surgery were assigned to observations, chemoradiotherapy (bolus 5-FU / split-course radiation), chemotherapy (5-FU / leucovorin), or chemoradiotherapy and chemotherapy. In this study a survival benefit for adjuvant chemotherapy not for chemoradiotherapy was observed and the Kaplan-Meier analysis showed a reduced survival rate following chemoradiotherapy compared to no chemoradiotherapy of $29 \%$ versus $41 \%$ patients respectively after 2 years, which was statistically significant. The impact of ESPAC-1 trial on therapeutic decisions in Europe were high, however substantial 
criticism have been also raised for this study. A meta-analysis data on 875 patients indicated a $25 \%$ significant reduction in the risk of death with chemotherapy. A median survival was estimated at 19 months with, and 13.5 months without, chemotherapy respectively [32]. In this meta-analysis no significant difference in the risk of death with chemoradiation to chemotherapy was observed and the median survival remained same (15.8 months with chemoradiation and 15.2 without). Still the authors concluded that the chemotherapy could be an effective adjuvant treatment for pancreatic cancer but not chemoradiation. Later several meta-analyses studies have confirmed survival benefit for adjuvant chemotherapy and no survival benefit for adjuvant chemoradiotherapy [33-36]. The meta analysis by Boeck et al, demonstrated that adjuvant chemotherapy provided a significant increase in median survival. Results from the Charite Onkologie (CONKO-001) trial have shown that adjuvant chemotherapy with gemcitabine confers about a 6-month improvement in median disease-free survival in patients with R0 and R1 resections. However; there was no statistically significant improvement in the overall survival between the gemcitabine and the observation groups [37,38]. For locally advanced and unresectable tumors, the choice is shown to be chemotherapy (5- Fluorouracil or gemcitabine) combined with radiotherapy, and followed by chemotherapy. This combined modality has been proven to prolong survival, and on occasion, results in regression of unresectable tumors to the point where salvage surgery may be performed [39]. However this concept of pre-operative radiochemotherapy is alluring and its effect for resectable cancer is not clear. The use of adjuvant chemotherapy showed a trend towards improved overall survival. For the patients who suffer from rapid progression and metastasis, gemcitabine chemotherapy alone or in combination with erlotinib is recommended. This treatment can be effective in helping to palliate symptoms of cancer and could confer a small but welcome survival advantage [40]. In the ESPAC-3 trial, 
the use of gemcitabine versus 5-FU demonstrated equivalent survival for both treatments, but more favorable safety profile with gemcitabine [41]. Gemcitabine monotherapy given weekly for three weeks (every 28 days) is the current standard of care, especially in patients with poor performance status. Still the average survival remains six months or less [42]. Combination chemotherapy with gemcitabine plus cisplatin, oxaliplatin, capecitabine, or irinotecan is usually reserved for patients with a good performance status due to the risk of undesirable side effects [43]. Patients who maintain a good performance status upon relapse may be treated with gemcitabine (if not previously used as a first-line treatment), oral capecitabine with or without oxaliplatin, or the FOLFIRINOX regimen (fluorouracil, leucovorin, irinotecan and oxaliplatin) [44,45]. In a recent clinical study, patients with advanced PC who received first-line chemotherapy with FOLFIRINOX lived longer than those who received gemcitabine alone. However, patients who received this combination had more side effects, and recommended that it should be used only for patients who are otherwise healthy despite the cancer [46].

The combination of gemcitabine plus erlotinib has recently received FDA approval for first-line treatment of metastatic PC (available at http://www.drugs.com/news/tarceva-approvedcombination-gemcitabine-pancreatic-cancer-1594.html). Erlotinib is an oral tyrosine kinase inhibitor and is known to inhibit the effects of epidermal growth factor receptor 1 (EGFR/HER1) pathway, known to be involved in the growth of cancers [47]. This combination offers a modest survival advantage (average of one month more) over gemcitabine alone. Although erlotinib is generally well tolerated, it can lead to the additional side effects of rash and diarrhea, which can be severe. Treatment involving erlotinib is cost prohibitive and clinicians and patients together must make the decision of the potential side effects and whether increased cost is worth the modest gains in survival. Although the rationale for adjuvant chemotherapy is sound, this 
approach remains controversial for most PC patients. Large, well-designed trials have reported conflicting results or shown only marginal success, making effective treatment of PC elusive.

\section{Developing therapies}

The major focus of research is on finding better treatments for PC. Apart from improvments in surgery and chemoradiotherapy the best combination treatments for people with advanced PC has not been achieved yet. Many clinical trials are being done to test new combinations of chemotherapy drugs for PC. Recently, Polaris Group announced that ADI-PEG 20 works synergistically with hydroxychloroquine (ChQ), an inhibitor of autophagy, in a human PC cell line. A majority of human pancreatic adenocarcinomas are deficient in argininosuccinate synthase (ASS) and are therefore sensitive to arginine deprivation by ADI-PEG 20. ASSdeficient cell lines undergo caspase-dependent cell death when deprived of arginine, but also undergo autophagy. ChQ at low doses inhibited autophagy induced by ADI-PEG 20, but increased cell death. In xenografts, addition of ChQ to a sub-therapeutic dose of ADI-PEG 20 increased tumor suppression, decreased autophagy and increased cell death compared to either treatment alone. Combining ChQ with ADI-PEG 20 in pancreatic cancer may increase the cell death induced by ADI-PEG 20 by blocking ADI-PEG 20-induced autophagy. ADI-PEG 20 is at the commencement phase II clinical studies, to investigate its role in suppressing PC (available at http://www.drugs.com/clinical_trials/cavatak-pancreatic-cancer-preclinical-research-

presentation-11169.html). Further the results from pre-clinical studies of triphendiol, a prodrug NV-143 under development by Marshall Edwards, demonstrates anti-proliferative activity in PC when used as both a monotherapy and as a chemosensitizer of gemcitabine (Gemzar ${ }^{\circledR}$, Eli Lilly and Company). Their studies show that pre-treatment with Triphendiol enhances the cytotoxic 
effect of gemcitabine and induces p53-independent G2/M cell cycle arrest and also causes the activation of the intrinsic apoptotic pathway in PC cells. Recently the Viralytics Limited has announced a Phase II trial using the oncolytic activity of Coxsackievirus A21 (CAVATAKTM). Their studies have indicated that CAVATAKTM uses the ICAM-1 receptor, highly expressed in human PC cells, and is able to specifically invade and destroy cancerous cells (available at http://www.drugs.com/clinical_trials/cavatak-pancreatic-cancer-preclinical-research- resentation11169.html). A Phase I trial of CEP-701, an inhibitor of tyrosine kinases including Flt-3, TRKA/B and JCK-2, with gemcitabine in patients with advanced adenocarcinoma of the pancreas also appears promising [48]. Further the hypoxia-activated prodrug, 3, 5-dinitrobenzamide nitrogen mustard PR-104, which recently entered clinical trials, was shown to have a greater killing potential of hypoxic cells (radioresistant) than aerobic cells in xenografts cell lines, and also showed that single-agent activity in six of eight xenograft models had greater than additive antitumor activity in combination with gemcitabine, which is likely to spare hypoxic cells [49]. In 2006 Mornex et al., [50] showed the feasibility of preoperative combined radiation therapy and chemotherapy with 5-fluorouracil and cisplatin in potentially resectable pancreatic adenocarcinoma without progression, thereby making this option clinically relevant. In all the aforementioned studies, very few patients were enrolled to limiting any significant evaluation of these drugs. Yet the large proportion of tumor progression in these patients during and after chemoradiation justifies the use of more efficient drugs, such as gemcitabine and optimized radiotherapy including sublethal doses of radiation in combination with molecular targeted therapy.

\section{Gene therapy}


The gene "targeted therapies" may provide another option for treating pancreatic cancer. The increasing knowledge of the aberrant activation of different signaling mechanisms and their molecules involved in cancer have contributed in understanding how PC cells are different from normal cells. Researchers have started developing newer drugs that could exploit these differences by attacking only specific targets. As we now know that overcoming resistance to chemotherapy is the greatest challenge for PC treatment. This challenge could be overcome by the use of a targeted approach. This therapy could be an effective strategy to revert chemoresistance, thereby increasing the efficacy of chemotherapy. Gene targeted therapies may prove to be useful along with, or instead of, current treatment regimens. In general, they seem to have fewer side effects than traditional chemotherapy drugs. Various molecular targets involved in pancreatic tumor development are being increasingly acknowledged and researched as contributors to chemoresistance in PC. Recently, studies on the role of human concentrative nucleoside transporter-1 (hCNT1), a high-affinity pyrimidine nucleoside transporter, was found to be involved in promoting the chemosensitivity of human PC cells to gemcitabine [51]. Compared to normal pancreas and pancreatic ductal epithelial cells, hCNT1 expression was frequently reduced in pancreatic tumors and tumor cell lines. Consequently, overexpression of hCNT1 could increase sensitivity to gemcitabine in pancreatic cells. Recent studies also demonstrated the role of Rho guanosine triphosphatases (GTPases) and their downstream signaling pathways as attractive targets for anticancer therapy [52]. RhoGTPases belong to a family of small proteins which function as molecular switches in a variety of signaling pathways following stimulation of cell surface receptors. RhoGTPases regulate numerous cellular processes including cytoskeleton organization, gene transcription, cell proliferation, migration, growth and cell survival. Because of their central role in regulating processes that are 
dysregulated in cancer, it seems reasonable to conclude that defects in the RhoGTPase pathway may be involved in the development of cancer. RhoGTPase activity is regulated by a number of protein families which include: guanine nucleotide exchange factors (GEFs), GTPase activating proteins (GAPs) and guanine nucleotide-dissociation inhibitors (GDIs). GDIs regulate Rho GTPase activity by inhibiting GDP dissociation, promoting the inactive form [53]. Studies found an association between elevated RhoGDI2 expression and the ability of PC cells to invade along nerve tracts [54]. Vav1 functions as a GEF for Rac1 GTPase following EGF stimulation and can bind to both RhoGDI2 and Rac in a complex, and RhoGDI2 increases Vav1's ability to activate NFAT [55]. Interestingly, ectopic expression of Vav1 has been detected in many human cancers including PC suggesting that it plays a significant role in the development and progression of human malignancies. Indeed, depletion of Vav1 expression in CFPAC and MiaPaCa2 PC cell lines resulted in the reduction of colony formation in soft agar in vitro and reduction of tumor size in nude mice [56]. Together, these findings indicate that Vav1 acts as an upstream activator of Rac1, RhoA and possibly Cdc42 signaling pathways in response to extracellular stimulation, Which leads to cytoskeleton changes and ultimately to increased cell motility. This makes Vav1 a potential target for PC. Similarily, Glycogen synthase kinase $3 \beta$ (GSK3 $\beta$ ), a serine/threonine protein kinase, which has a well characterized roles in the regulation of gene transcription and in oncogenic signaling, has been proposed as a potential therapeutic target for PC [57]. Apart from PC, deregulated GSK3 $\beta$ promotes liver cancers and glioblastomas [57]. Furthermore, inhibition of GSK3 $\beta$ attenuates PC cells survival and proliferation, induces cell senescence and apoptosis and sensitizes the tumor cells to chemotherapeutic agents and ionizing radiation. Yet another emerging cancer target is the nonreceptor tyrosine kinase, c-Src, which is shown to be overexpressed in PC [58]. Src tyrosine kinase activity is important in the epithelial to 
mesenchymal transition that occurs in the early stages of carcinoma cell invasion, and its role has also been implicated in angiogenesis and vascular permeability [59,60]. Further, studies to investigate the in vivo efficacy and pharmacodynamic effects of bosutinib (SKI-606), a Src/Abl inhibitor, using a panel of human pancreatic tumor xenografts showed sensitization of $20 \%$ of tumors to bosutinib. In sensitive tumors, bosutinib resulted in increased apoptosis, decreased phosphorylation of key signaling molecules downstream of Src, defined tumor growth of $<45 \%$ that of control tumors [61], therby suggesting the role bosutinib and other Src inhibitors in clinical development of PC. Carcinoembryonic antigen-related cell adhesion molecule (CEACAM) 6 has also been reported as a potential biomarker and therapy target for pancreatic adenocarcinoma [62]. Robust expression of CEACAM6 was found in $>90 \%$ of invasive pancreatic adenocarcinomas as well as in intraepithelial neoplastic lesions [63]. Its potential therapy target has been evaluated by an antibody-drug conjugate (ADC). The anti-CEACAM6 ADC showed efficacy against established CEACAM6-expressing tumors. Functionally, CEACAM6 has been implicated in cell adhesion [64], cellular invasiveness [65], resistance to anoikis [66], and metastatic behavior of tumor cells [67], which are the properties that have been previously exploited by the investigators for various CEACAM6- targeted therapy approaches [68]. Studies on the genome-wide gene expression profiles of microdissected pancreatic ductal adenocarcinoma (PDAC) cells have identified a novel gene C2orf18, termed ANT2-binding protein (ANT2BP), as a potential molecular target for PDAC treatment [69]. Transcriptional and immunohistochemical analysis validated its overexpression in PDAC cells and limited expression in normal adult organs. Knockdown of C2orf18 by small-interfering RNA in PDAC cell lines resulted in induction of apoptosis and suppression of cancer cell growth, suggesting its essential role in maintaining viability of PDAC cells. Recently, our lab studies have shown that 
sh-RNA mediated simultaneous downregulation of urokinase-type plasminogen activator receptor and urokinase-type plasminogen activator (uPAR-uPA) retards in vivo tumor development in PC cells [70]. We also showed that MIA PaCa-2 and PANC-1 cells downregulated for both uPAR and uPA decreased angiogenesis. Further angiogenesis antibody array analysis demonstrated that the simultaneous downregulation of UPAR and UPA resulted in the downregulation of angiogenin and overexpression of RANTES. Earlier studies have shown the importance of urokinase-type plasminogen activator (uPA) and fibroblastic uPA receptor (uPAR) in liver metastasis of human PC [71] and also in migration [72]. Moreover, invasion studies suggest that uPAR is a potential target for therapy in patients with PC $[72,73]$. The mRNA expression of 15 genes from epidermal growth factor receptor (EGFR), insulin-like growth factor (IGF), and uPA families measured in 46 PDAC tissue samples using quantitative real-time reverse transcription-polymerase chain reaction, showed that of all the genes, the uPA/uPAR system had a greater influence on survival of PDAC [74]. This finding highlights the importance and the relevance of specifically targeting the uPA-uPAR system. The suppression of extracellular matrix (ECM) degrading proteases such as urokinase-type plasminogen activator (uPA) and matrix metalloproteinases (MMPs) such as MMP-9 and MMP-2 have shown significant decrease in the tumor-related processes, including adhesion, migration, proliferation and angiogenesis in different cancers [75-78] Studies have also reported that MMP-9 and MMP2 are involved in promoting PC growth and thus their inhibitors are potentially useful in the treatment against PC [79].

\section{Developing strategies and new approaches to treatment}


Despite the emergence of new molecular targets and the use of various therapeutic combinations, none of the treatment options available are curative in patients with advanced cancer. A growing body of evidence is supporting the idea that human cancers can be considered as a stem cell disease. Malignancies are believed to originate from a pluripotent subset of cells that have the capacity to seed the cellular heterogeneity seen in tumors [80]. The hypothesis of stem celldriven tumorigenesis raises questions as to whether the current treatment strategies, most of which target rapidly dividing cells, are able to efficiently target these cancer stem cells that are slow-cycling. Recent characterization of these cancer stem cells have led to the identification of key signaling pathways making it possible for cancer stem cells targets for therapeutic interventions. Their potential targets include drug-effluxing capabilities, anti-apoptotic mechanisms, and induction of differentiation [81]. In lines of this approach recently, an adoptive T-cell transfer approach was developed to target pancreatic tumors expressing prostate stem cell antigen (PSCA). This is a promising novel treatment for patients with PC as the antigen PSCA, is frequently expressed on almost all PC cells and can be a target for immune-mediated therapy using chimeric (CAR)-modified antigen receptor [82]. Cancer stem cells (CSCs) proliferate extensively, self-renew, express drug-resistant and also express anti-apoptotic proteins, these aspects make the CSC difficult to target. A study on resveratrol (3,5,4'-trihydroxy-trans-stilbene) and its role in inhibiting the self-renewal capacity of pancreatic CSCs derived from human primary tumors and from Kras (G12D) mice and showed that resveratrol induces apoptosis by activating capase-3/7 and inhibiting the expression of Bcl-2 and XIAP in human CSCs. It also inhibits pluripotency maintaining factors (Nanog, Sox-2, c-Myc and Oct-4) and the drugresistance gene, ABCG2, in CSCs [83].. Another study of human PC revealed that a specific subpopulation of cancer cells (CSCs) which represents $0.5 \%$ to $1.0 \%$ of all PC cells, express cell 
surface markers such as CD44, CD24, ESA and ALDH1 and upregulate important developmental genes that maintain self-renewal in normal stem cells which includes Sonic hedgehog (Shh) and BMI-1 [84]. The aberrantly activated Hedgehog (Hh) pathway has been shown to play a crucial role in the initiation and maintenance of pancreatic neoplasia and is known to contribute to chemoresistance [85-87]. Further, a combination of agents that target non-overlapping mechanisms such as histone deacetylases (HDACs) and hedgehog (Hh) signaling was shown to be an effective strategy to suppress the growth of gemcitabine-resistant pancreatic adenocarcinoma cells [88]. Recently we identified that Lhx2, a transcription factor required to drive activated stem cells toward terminal differentiation, and a molecule upstream of signals such as Wnt/ $\beta$-catenin, Bmp, and Shh [89] interacts with uPA in the nucleus [70]. It is worth noting that the overexpression of uPA is at the invasive fronts of most cancer, and further that its association with stem cell markers provides a basis for considering the uPAR-uPA system as potential therapeutic target for the treatment of PC (Figure 1). The understanding of various signaling systems such as proteases involved in cancer progression can enable the better understanding and formulation of strategies to target the CSC and once such system, the uPA/uPAR system is a good candidate for targeting CSC [70,90].

Along with proteases the microRNAs are increasingly being acknowledged as useful biomarkers for classifying cancers and for providing information on their chemo- and radiosensitivity. MicroRNAs (miRNA) have emerged as a critical class of negative regulators of gene expression through modulation of post-transcriptional activity of multiple target mRNAs by repression of translation or direct cleavage [91]. The role of miRNAs in proliferation, differentiation and 
apoptosis is now being identified. The presence of these miRNA genes at sites of translocation breakpoints or deletions results in the aberrant expression of not only these miRNAs but also of their target genes [83], which results in unregulated growth and proliferation. Furthermore, over the last few years miRNAs have emerged as a prominent class of gene regulators and their aberrant expression has been linked to different tumors, including PDAC [92-94]. Expression profiling has identified several miRNAs that are aberrantly expressed in PDAC, including four miRNAs which are differentially expressed in other tumors including miR-155, miR-21, miR221 and miR-222 [94]. These results were corroborated by other studies showing that miR-21 was among the top miRNA with increased expression in PDAC [92,95*]. In contrast, transfection of miR-21 precursors in cancer cells stimulated invasion, extravasation, and metastasis in in vivo models of glioma, colorectal, and breast cancer [96,97] as well as in vitro models of PDAC [98]. High miR-21 expression was associated with a more aggressive pancreatic endocrine tumor, characterized by increased Ki67 proliferation index and liver metastasis [99]. In particular, inhibition of miR-21 increased sensitivity to gemcitabine in cholangiocarcinoma and PDAC cells $[98,100,101]$. MiR-21 leads to downregulation of PTEN and activation of the PI3K/Akt/mTOR pathway, rendering the cancer cells less susceptible to apoptosis [102]. Another recently identified miR-21 target is RECK [103]. These factor make miR-21 a potential therapeutic target for PC. Further, microarray analysis has shown that miRNAs are also associated with PC stem cells [102]. Recently miR-10b was shown to be a novel marker for assessing suspicious pancreatic lesions and its expression was also shown to be associated with improved response to multimodality neoadjuvant therapy, longer time to metastasis and improved overall survival [104]. Thus targeting relevant miRNAs either by antagomirs or by overexpression has potential therapeutic relevance. 


\section{Expert's opinion}

We have seen that the current chemotherapy strategy (gemcitabine) has very little benefit to PC patients. Unfortunately due to late presentation, delayed diagnosis and no effective treatment, death is inevitable. There is a need to combine numerous strategies and adapt the sensitivity of PC cells to the available drugs without affecting normal cells. An ideal therapeutic strategy could be developed by combining surgery and or chemo/radiotherapy with molecular targeted therapy that can be effective in multiple patient populations. The development of therapies targeting chemoresistance should enable the quick increase in survival rate of PC patients. Growth factor inhibitors that target epidermal growth factor receptor (EGFR) are now also being studied and one among them is erlotinib (Tarceva), which is already approved for use along with gemcitabine. Research in our laboratory focuses on the uPA/uPAR system which appears to play a major role in the invasive processes underlying cancer spread and metastasis. We showed that shRNA mediated downregulation of uPA/uPAR inhibits invasion, migration and angiogenesis of PC and increases chemo and radiosensitivity (Unpublished data). uPA is an attractive target because its expression is generally restricted to tumor tissue and is not usually expressed by cells outside the tumor microenvironment. However, uPAR may be up-regulated in some cells during non-cancerous processes such as wound healing and in response to infection. An antibody known as, ATN-658 is currently in preclinical development for uPA targeted therapy for cancer. Further on going research work on the peptide inhibitors and small molecule inhibitors targeting uPAuPAR system has shown promising results in animal models of PC but none of them have reached the preclinical or clinical stage. A further understanding of the molecular mechanisms will enable the development of robust therapies targeting cancer stem cells which are thought to contribute to chemo and radioresistance. The frequent reccurrence of cancer after conventional 
treatments such as surgery, radiation and/or chemotherapy results because the basic underlying cause of the cancer are rarely considered and consequently remains uncorrected. Treatment of PC may need to be individualized as each patient's disease may be different from another. Cancer as such is not one disease but a collection of many similar diseases.

\section{Conclusion}

PC remains one of the most difficult human malignancies to treat. A systemic therapy improving the overall survival of patients with advanced PC is still missing. Untill today PC remains a highly chemoresistant malignancy that urgently requires new therapeutic targets as well as robust predictive markers of sufficient sensitivity to existing regimens. The treatment of patients with advanced PC failing to respond to gemcitabine monotherapy remains a true challenge as no established second-line treatment exists. Therefore, it seems imperative that identifying new targets and targeting multiple genes/molecular pathways related to chemoresistance should be the final line of treatment. Hopefully, an integrated molecular-chemical approach that targets multiple regulatory checkpoints will be successful in combating this deadly cancer. 


\section{Reference List}

1. Ghadirian P, Lynch HT, Krewski D. Epidemiology of pancreatic cancer: an overview. Cancer Detect Prev 2003;27:87-93

2. Inoue M, Yamamoto S, Kurahashi N, et al. Daily total physical activity level and total cancer risk in men and women: results from a large-scale population-based cohort study in Japan. Am J Epidemiol 2008;168:391-403

3. Mysliwiec P, Kedra B. Causes of delayed diagnosis of pancreatic cancer. Own study and proposed algorithm. Przegl Lek 2008;65:345-8

4. Dhayat S, Mardin WA, Mees ST, Haier J. Epigenetic markers for chemosensitivity and chemoresistance in pancreatic cancer--a review. Int J Cancer 2011;129:1031-41

5. Tranah GJ, Holly EA, Wang F, Bracci PM. Cigarette, cigar and pipe smoking, passive smoke exposure, and risk of pancreatic cancer: a population-based study in the San Francisco Bay Area. BMC Cancer 2011;11:138.:138

6. Lowenfels AB, Maisonneuve P, DiMagno EP, et al. Hereditary pancreatitis and the risk of pancreatic cancer. International Hereditary Pancreatitis Study Group. J Natl Cancer Inst 1997;89:442-6

7. Danes BS, Lynch HT. A familial aggregation of pancreatic cancer. An in vitro study. JAMA 1982;247:2798-802 
8. Lynch HT, Fitzsimmons ML, Smyrk TC, et al. Familial pancreatic cancer: clinicopathologic study of 18 nuclear families. Am J Gastroenterol 1990;85:54-60

9. Calle EE, Kaaks R. Overweight, obesity and cancer: epidemiological evidence and proposed mechanisms. Nat Rev Cancer 2004;4:579-91

10. Cowgill SM, Muscarella P. The genetics of pancreatic cancer. Am J Surg 2003;186:27986

11. Lochan R, Daly AK, Reeves HL, Charnley RM. Family history of cancer and tobacco exposure in index cases of pancreatic ductal adenocarcinoma. $\mathrm{J}$ Oncol 2011;2011:215985-E-pub ahead of print

12. Shi C, Hruban RH, Klein AP. Familial pancreatic cancer. Arch Pathol Lab Med 2009;133:365-74

13. Grover S, Syngal S. Hereditary pancreatic cancer. Gastroenterology 2010;139:1076-80, 1080

14. Kastrinos F, Mukherjee B, Tayob N, et al. Risk of pancreatic cancer in families with Lynch syndrome. JAMA 2009;302:1790-5

15. Kluijt I, Cats A, Fockens P, et al. Atypical familial presentation of FAMMM syndrome with a high incidence of pancreatic cancer: case finding of asymptomatic individuals by EUS surveillance. J Clin Gastroenterol 2009;43:853-7 
16. Naderi A, Couch FJ. BRCA2 and pancreatic cancer. Int J Gastrointest Cancer 2002;31:99-106

17. Tokura M, Kamisawa T, Kuwata G, et al. Pancreatic cancer as the fourth cancer in a patient with Peutz-Jeghers syndrome and a history of familial pancreatic cancer. Pancreas 2011;40:169-70

18. Abou-Alfa GK, Chapman PB, Feilchenfeldt J, et al. Targeting mutated K-ras in pancreatic adenocarcinoma using an adjuvant vaccine. Am J Clin Oncol 2011;34:321-5

19. Brody JR, Costantino CL, Potoczek M, et al. Adenosquamous carcinoma of the pancreas harbors KRAS2, DPC4 and TP53 molecular alterations similar to pancreatic ductal adenocarcinoma. Mod Pathol 2009;22:651-9

20. Li J, Weghorst CM, Tsutsumi M, et al. Frequent p16INK4A/CDKN2A alterations in chemically induced Syrian golden hamster pancreatic tumors. Carcinogenesis $2004 ; 25: 263-8$

21. Grant WB, Mohr SB. Ecological studies of ultraviolet B, vitamin D and cancer since 2000. Ann Epidemiol 2009;19:446-54

22. Kinoshita S, Wagatsuma Y, Okada M. Geographical distribution for malignant neoplasm of the pancreas in relation to selected climatic factors in Japan. Int J Health Geogr 2007;6:34.:34

23. Raimondi S, Maisonneuve P, Lowenfels AB. Epidemiology of pancreatic cancer: an overview. Nat Rev Gastroenterol Hepatol 2009;6:699-708 
24. Rosewicz S, Wiedenmann B. Pancreatic carcinoma. Lancet 1997;349:485-9

25. Long J, Zhang Y, Yu X, et al. Overcoming drug resistance in pancreatic cancer. Expert Opin Ther Targets 2011;15:817-28

26. Lockhart AC, Rothenberg ML, Berlin JD. Treatment for pancreatic cancer: current therapy and continued progress. Gastroenterology 2005;128:1642-54

27. Meriggi F, Forni E. Extended lymphadenectomy for carcinoma of pancreatic head. Personal experience. G Chir 2002;23:383-90

28. Yeo CJ, Abrams RA, Grochow LB, et al. Pancreaticoduodenectomy for pancreatic adenocarcinoma: postoperative adjuvant chemoradiation improves survival. A prospective, single-institution experience. Ann Surg 1997;225:621-33

29. Kalser MH, Ellenberg SS. Pancreatic cancer. Adjuvant combined radiation and chemotherapy following curative resection. Arch Surg 1985;120:899-903

30. Neoptolemos JP, Raraty MG, Ghaneh P, et al. Adjuvant and additive therapy for cancer of the pancreas. Chirurg 2003;74:191-201

31. Neoptolemos JP, Stocken DD, Dunn JA, et al. Influence of resection margins on survival for patients with pancreatic cancer treated by adjuvant chemoradiation and/or chemotherapy in the ESPAC-1 randomized controlled trial. Ann Surg 2001;234:758-68

32. Stocken DD, Buchler MW, Dervenis C, et al. Meta-analysis of randomised adjuvant therapy trials for pancreatic cancer. Br J Cancer 2005;92:1372-81 
33. Boeck S, Ankerst DP, Heinemann V. The role of adjuvant chemotherapy for patients with resected pancreatic cancer: systematic review of randomized controlled trials and metaanalysis. Oncology 2007; 72:314-21

34. Drudi F, Tassinari DCC, Carloni FSC, et al. Adjuvant treatments in pancreatic cancer: Preliminary data of a pooled analysis. J Clin Oncol 29(Suppl.) . 2011. Abstract \#4042.

35. Khanna A, Walker GR, Livingstone AS, et al. Is adjuvant 5-FU-based chemoradiotherapy for resectable pancreatic adenocarcinoma beneficial? A meta-analysis of an unanswered question. J Gastrointest Surg 2006;10:689-97

36. Neoptolemos JP, Stocken DD, Tudur SC, et al. Adjuvant 5-fluorouracil and folinic acid vs observation for pancreatic cancer: composite data from the ESPAC-1 and -3(v1) trials. Br J Cancer 2009;100:246-50

37. Oettle $\mathrm{H}$, Post $\mathrm{S}$, Neuhaus $\mathrm{P}$, et al. Adjuvant chemotherapy with gemcitabine vs observation in patients undergoing curative-intent resection of pancreatic cancer: a randomized controlled trial. JAMA 2007;297:267-77

38. Richter J, Saif MW. Updates in adjuvant therapy in pancreatic cancer: gemcitabine and beyond. Highlights from the "2010 ASCO Gastrointestinal Cancers Symposium". Orlando, FL, USA. January 22-24, 2010. JOP 2010;11:144-7

39. Sawaki A, Hoki N, Ito S, et al. Clinical impact of radiotherapy for locally advanced pancreatic cancer. J Gastroenterol 2009;44:1209-14 
40. Moore MJ, Goldstein D, Hamm J, et al. Erlotinib plus gemcitabine compared with gemcitabine alone in patients with advanced pancreatic cancer: a phase III trial of the National Cancer Institute of Canada Clinical Trials Group. J Clin Oncol 2007;25:1960-6

41. Neoptolemos JP, Stocken DD, Bassi C, et al. Adjuvant chemotherapy with fluorouracil plus folinic acid vs gemcitabine following pancreatic cancer resection: a randomized controlled trial. JAMA 2010;304:1073-81

42. Hirao K, Kawamoto H, Sakakihara I, et al. A 4-week versus a 3-week schedule of gemcitabine monotherapy for advanced pancreatic cancer: a randomized phase II study to evaluate toxicity and dose intensity. Int J Clin Oncol 2011;

43. Gong JF, Zhang XD, Li J, et al. [Efficacy of gemcitabine-based chemotherapy on advanced pancreatic cancer]. Ai Zheng 2007;26:890-4 ;16(6):637-45

44. Assaf E, Verlinde-Carvalho M, Delbaldo C, et al. 5-Fluorouracil/Leucovorin Combined with Irinotecan and Oxaliplatin (FOLFIRINOX) as Second-Line Chemotherapy in Patients with Metastatic Pancreatic Adenocarcinoma. Oncology 2011;80:301-6

45. Lowery MA, O'Reilly EM. New approaches to the treatment of pancreatic cancer: from tumor-directed therapy to immunotherapy. BioDrugs 2011;25:207-16

46. Conroy T, Desseigne F, Ychou M, et al. FOLFIRINOX versus gemcitabine for metastatic pancreatic cancer. N Engl J Med 2011;364:1817-25 
47. Oberstein PE, Saif MW. First-line treatment for advanced pancreatic cancer. Highlights from the "2011 ASCO Gastrointestinal Cancers Symposium". San Francisco, CA, USA. January 20-22, 2011. JOP 2011;12:96-100

48. Chan E, Mulkerin D, Rothenberg M, et al. A phase I trial of CEP-701 + gemcitabine in patients with advanced adenocarcinoma of the pancreas. Invest New Drugs 2008;26:2417

49. Patterson AV, Ferry DM, Edmunds SJ, et al. Mechanism of action and preclinical antitumor activity of the novel hypoxia-activated DNA cross-linking agent PR-104. Clin Cancer Res 2007;13:3922-32

50. Mornex F, Girard N, Scoazec JY, et al. Feasibility of preoperative combined radiation therapy and chemotherapy with 5-fluorouracil and cisplatin in potentially resectable pancreatic adenocarcinoma: The French SFRO-FFCD 97-04 Phase II trial. Int J Radiat Oncol Biol Phys 2006;65:1471-8

51. Bhutia YD, Hung SW, Patel B, et al. CNT1 expression influences proliferation and chemosensitivity in drug-resistant pancreatic cancer cells. Cancer Res 2011;71:1825-35

52. Sahai E, Marshall CJ. RHO-GTPases and cancer. Nat Rev Cancer 2002;2:133-42

53. DerMardirossian C, Bokoch GM. GDIs: central regulatory molecules in Rho GTPase activation. Trends Cell Biol 2005;15:356-63 
54. Koide N, Yamada T, Shibata R, et al. Establishment of perineural invasion models and analysis of gene expression revealed an invariant chain (CD74) as a possible molecule involved in perineural invasion in pancreatic cancer. Clin Cancer Res 2006;12:2419-26

55. Groysman M, Hornstein I, Alcover A, Katzav S. Vav1 and Ly-GDI two regulators of Rho GTPases, function cooperatively as signal transducers in $\mathrm{T}$ cell antigen receptor-induced pathways. J Biol Chem 2002;277:50121-30

56. Fernandez-Zapico ME, Gonzalez-Paz NC, Weiss E, et al. Ectopic expression of VAV1 reveals an unexpected role in pancreatic cancer tumorigenesis. Cancer Cell 2005;7:39-49

57. Miyashita K, Nakada M, Shakoori A, et al. An emerging strategy for cancer treatment targeting aberrant glycogen synthase kinase 3 beta. Anticancer Agents Med Chem 2009;9:1114-22

58. Hilbig A. Src kinase and pancreatic cancer. Recent Results Cancer Res 2008;177:17985.:179-85

59. Boyer B, Valles AM, Edme N. Induction and regulation of epithelial-mesenchymal transitions. Biochem Pharmacol 2000;60:1091-9

60. Park SI, Shah AN, Zhang J, Gallick GE. Regulation of angiogenesis and vascular permeability by Src family kinases: opportunities for therapeutic treatment of solid tumors. Expert Opin Ther Targets 2007;11:1207-17 
61. Messersmith WA, Rajeshkumar NV, Tan AC, et al. Efficacy and pharmacodynamic effects of bosutinib (SKI-606), a Src/Abl inhibitor, in freshly generated human pancreas cancer xenografts. Mol Cancer Ther 2009;8:1484-93

62. Duxbury MS, Ito $\mathrm{H}$, Benoit E, et al. CEACAM6 is a determinant of pancreatic adenocarcinoma cellular invasiveness. Br J Cancer 2004;91:1384-90

63. Strickland LA, Ross J, Williams S, et al. Preclinical evaluation of carcinoembryonic cell adhesion molecule (CEACAM) 6 as potential therapy target for pancreatic adenocarcinoma. J Pathol 2009;218:380-90

64. Duxbury MS, Ito H, Ashley SW, Whang EE. c-Src-dependent cross-talk between CEACAM6 and alphavbeta3 integrin enhances pancreatic adenocarcinoma cell adhesion to extracellular matrix components. Biochem Biophys Res Commun 2004;317:133-41

65. Duxbury MS, Ito H, Benoit E, et al. Overexpression of CEACAM6 promotes insulin-like growth factor I-induced pancreatic adenocarcinoma cellular invasiveness. Oncogene 2004;23:5834-42

66. Duxbury MS, Ito H, Benoit E, et al. A novel role for carcinoembryonic antigen-related cell adhesion molecule 6 as a determinant of gemcitabine chemoresistance in pancreatic adenocarcinoma cells. Cancer Res 2004;64:3987-93

67. Duxbury MS, Ito H, Zinner MJ, et al. CEACAM6 gene silencing impairs anoikis resistance and in vivo metastatic ability of pancreatic adenocarcinoma cells. Oncogene 2004;23:465-73 
68. Duxbury MS, Ito H, Ashley SW, Whang EE. CEACAM6 as a novel target for indirect type 1 immunotoxin-based therapy in pancreatic adenocarcinoma. Biochem Biophys Res Commun 2004;317:837-43

69. Kashiwaya K, Hosokawa M, Eguchi H, et al. Identification of C2orf18, termed ANT2BP (ANT2-binding protein), as one of the key molecules involved in pancreatic carcinogenesis. Cancer Sci 2009;100:457-64

70. Gorantla B, Asuthkar S, Rao JS, et al. Suppression of the uPAR-uPA System Retards Angiogenesis, Invasion, and In Vivo Tumor Development in Pancreatic Cancer Cells. Mol Cancer Res 2011;9:377-89

71. He Y, Liu XD, Chen ZY, et al. Interaction between cancer cells and stromal fibroblasts is required for activation of the uPAR-uPA-MMP-2 cascade in pancreatic cancer metastasis. Clin Cancer Res 2007;13:3115-24

72. Bauer TW, Liu W, Fan F, et al. Targeting of urokinase plasminogen activator receptor in human pancreatic carcinoma cells inhibits c-Met- and insulin-like growth factor-I receptor-mediated migration and invasion and orthotopic tumor growth in mice. Cancer Res 2005;65:7775-81

73. Hildenbrand R, Niedergethmann M, Marx A, et al. Amplification of the urokinase-type plasminogen activator receptor (uPAR) gene in ductal pancreatic carcinomas identifies a clinically high-risk group. Am J Pathol 2009;174:2246-53 
74. Xue A, Scarlett CJ, Jackson CJ, et al. Prognostic significance of growth factors and the urokinase-type plasminogen activator system in pancreatic ductal adenocarcinoma. Pancreas 2008;36:160-7

75. Chandrasekar N, Mohanam S, Gujrati M, et al. Downregulation of uPA inhibits migration and PI3k/Akt signaling in glioblastoma cells. Oncogene 2003;22:392-400

76. Huang GJ, Yang CM, Chang YS, et al. Hispolon suppresses SK-Hep1 human hepatoma cell metastasis by inhibiting matrix metalloproteinase-2/9 and urokinase-plasminogen activator through the PI3K/Akt and ERK signaling pathways. J Agric Food Chem 2010;58:9468-75

77. Huang HY, Jiang ZF, Li QX, et al. Inhibition of human breast cancer cell invasion by siRNA against urokinase-type plasminogen activator. Cancer Invest 2010;28:689-97

78. Lai KC, Huang AC, Hsu SC, et al. Benzyl isothiocyanate (BITC) inhibits migration and invasion of human colon cancer HT29 cells by inhibiting matrix metalloproteinase-2/-9 and urokinase plasminogen (UPA) through PKC and MAPK signaling pathway. J Agric Food Chem 2010;58:2935-42

79. Uchima Y, Sawada T, Hirakawa K. Action of antiproteases on pancreatic cancer cells. JOP 2007;8:479-87

80. Clarke MF, Fuller M. Stem cells and cancer: two faces of eve. Cell 2006;124:1111-5

81. Subramaniam D, Ramalingam S, Houchen CW, Anant S. Cancer stem cells: a novel paradigm for cancer prevention and treatment. Mini Rev Med Chem 2010;10:359-71 
82. Katari UL, Keirnan JM, Worth AC, et al. Engineered $\mathrm{T}$ cells for pancreatic cancer treatment. HPB (Oxford) 2011;13:643-50

83. Shankar S, Nall D, Tang SN, et al. Resveratrol inhibits pancreatic cancer stem cell characteristics in human and KrasG12D transgenic mice by inhibiting pluripotency maintaining factors and epithelial-mesenchymal transition. PLoS One 2011;6:e16530

84. Lee CJ, Dosch J, Simeone DM. Pancreatic cancer stem cells. J Clin Oncol 2008;26:280612

85. Lou H, Dean M. Targeted therapy for cancer stem cells: the patched pathway and ABC transporters. Oncogene 2007;26:1357-60

86. Morton JP, Mongeau ME, Klimstra DS, et al. Sonic hedgehog acts at multiple stages during pancreatic tumorigenesis. Proc Natl Acad Sci U S A 2007;104:5103-8

87. Thayer SP, di Magliano MP, Heiser PW, et al. Hedgehog is an early and late mediator of pancreatic cancer tumorigenesis. Nature 2003;425:851-6

88. Chun SG, Zhou W, Yee NS. Combined targeting of histone deacetylases and hedgehog signaling enhances cytoxicity in pancreatic cancer. Cancer Biol Ther 2009;8:1328-39

89. Tiede S, Paus R. Lhx2--decisive role in epithelial stem cell maintenance, or just the "tip of the iceberg"? Bioessays 2006;28:1157-60 
90. Gutova M, Najbauer J, Frank RT, et al. Urokinase plasminogen activator and urokinase plasminogen activator receptor mediate human stem cell tropism to malignant solid tumors. Stem Cells 2008;26:1406-13

91. Carthew RW, Sontheimer EJ. Origins and Mechanisms of miRNAs and siRNAs. Cell 2009;136:642-55

92. Bloomston M, Frankel WL, Petrocca F, et al. MicroRNA expression patterns to differentiate pancreatic adenocarcinoma from normal pancreas and chronic pancreatitis. JAMA 2007;297:1901-8

93. Iorio MV, Croce CM. MicroRNAs in cancer: small molecules with a huge impact. J Clin Oncol 2009;27:5848-56

94. Lee EJ, Gusev Y, Jiang J, et al. Expression profiling identifies microRNA signature in pancreatic cancer. Int J Cancer 2007;120:1046-54

*95. Szafranska AE, Doleshal M, Edmunds HS, et al. Analysis of microRNAs in pancreatic fine-needle aspirates can classify benign and malignant tissues. Clin Chem 2008;54:1716-24

96. Asangani IA, Rasheed SA, Nikolova DA, et al. MicroRNA-21 (miR-21) posttranscriptionally downregulates tumor suppressor Pdcd4 and stimulates invasion, intravasation and metastasis in colorectal cancer. Oncogene 2008;27:2128-36

97. Gabriely G, Wurdinger T, Kesari S, et al. MicroRNA 21 promotes glioma invasion by targeting matrix metalloproteinase regulators. Mol Cell Biol 2008;28:5369-80 
98. Moriyama T, Ohuchida K, Mizumoto K, et al. MicrRNA-21 modulates biological functions of pancreatic cancer cells including their proliferation, invasion, and chemoresistance. Mol Cancer Ther 2009;8:1067-74

99. Roldo C, Missiaglia E, Hagan JP, et al. MicroRNA expression abnormalities in pancreatic endocrine and acinar tumors are associated with distinctive pathologic features and clinical behavior. J Clin Oncol 2006;24:4677-84

100. Meng F, Henson R, Lang M, et al. Involvement of human micro-RNA in growth and response to chemotherapy in human cholangiocarcinoma cell lines. Gastroenterology 2006;130:2113-29

101. Park JK, Lee EJ, Esau C, Schmittgen TD. Antisense inhibition of microRNA-21 or -221 arrests cell cycle, induces apoptosis, and sensitizes the effects of gemcitabine in pancreatic adenocarcinoma. Pancreas 2009;38:e190-e199

102. Jung DE, Wen J, Oh T, Song SY. Differentially Expressed MicroRNAs in Pancreatic Cancer Stem Cells. Pancreas 2011;E-pub ahead of print

103. Krichevsky AM, Gabriely G. miR-21: a small multi-faceted RNA. J Cell Mol Med 2009;13:39-53

104. Preis M, Gardner TB, Gordon SR, et al. MicroRNA-10b Expression Correlates with Response to Neoadjuvant Therapy and Survival in Pancreatic Ductal Adenocarcinoma. Clin Cancer Res 2011;17:5812-21 


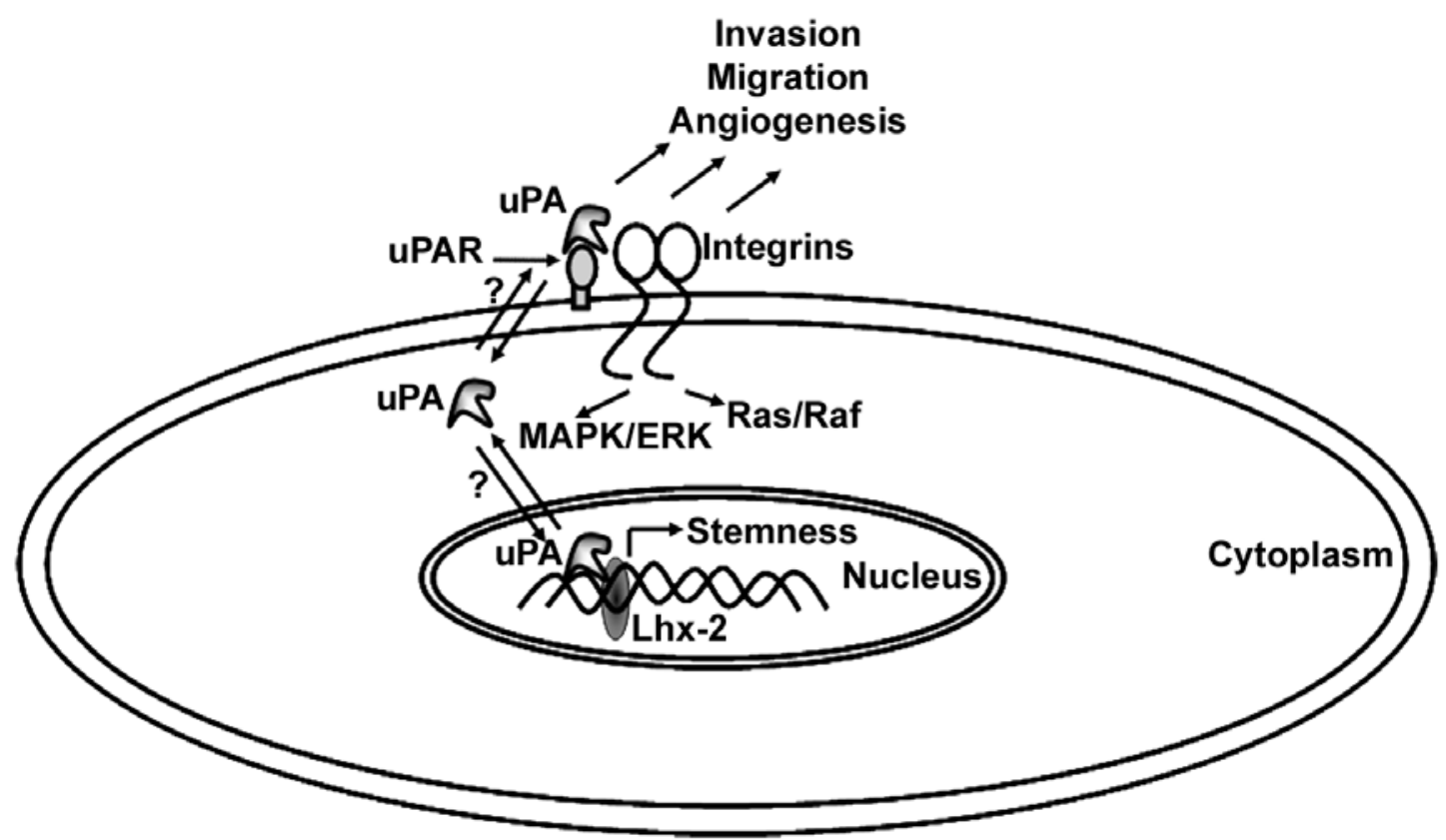

\section{Legends}

Figure 1 The uPA/uPAR system and its role in cancer stemness. The overexpression and activation of uPA-uPAR system in most cancer types and its further association with transcription factor such as Lhx-2, provides a basis for considering this system as a potential therapeutic target for the treatment of pancreatic cancer. 